ORIGINAL ARTICLE

\title{
Working conditions and fatigue in professional truck drivers at Israeli ports
}

\author{
S Sabbagh-Ehrlich, L Friedman, E D Richter
}

Injury Prevention 2005;11:110-114. doi: 10.1136/ip.2004.007682

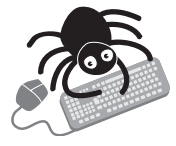

Figure 2 can be viewed on our website.

See end of article for authors' affiliations

....................

Correspondence to: Dr E Richter, Center for Injury Prevention, Unit of Occupational and Environmental Medicine, Hebrew UniversityHadassah Medical School, Jerusalem 91120, Israel; elir@cc.huji.ac.il
Background: Trucks represent $6 \%$ of all vehicles, but truck crashes account for $20 \%$ of road deaths in Israel, even though travel distances are usually short $(<200 \mathrm{~km})$ and overnight travel is uncommon.

Objective: To determine occupational and individual predictors of fatigue, falling asleep at the wheel, and involvement in crashes with injuries and deaths in truck drivers.

Setting and methods: We carried out field interviews of 160 port truck drivers regarding driver characteristics, workplace and driving conditions, employer-employee relations, medical conditions, sleep quality and fatigue, falling asleep at the wheel, and involvement in road crashes.

Results: One day before interview, 38.1\% of the drivers had worked more than the 12 hour legal limit. More than 30\% reported falling asleep at the wheel recently, and 13\% had prior involvement in a sleep related crash. Sixty seven $(41.9 \%)$ drivers said that their employer forced them to work beyond the legal 12 hour daily limit. Involvement in a crash with casualties was associated with poor sleep quality (adjusted $O R=2.9 ; p=0.042)$ and frequent difficulty finding parking when tired $(O R=3.7 ; p=0.049)$. Self assessment of fatigue underestimated fatigue from the Pittsburgh Sleep Quality Questionnaire. However fatigue occurred in many drivers without sleep problems and many crashes occurred without fatigue.

Conclusions: Prevention requires measures to reduce work stresses, screening drivers, speed control, and modal shifts. The work risks and adverse outcomes of truck drivers in large countries with long overnight journeys occur in a small country with small distances, relatively short work journeys, and little overnight travel.
$\mathrm{T}$ here is much interest in the contribution of fatigue to the high risks for job related illnesses and injuries of truck drivers, especially in long haul carriers. The California Highway Patrol reported that fatigue was a cause in $67 \%$ of truck crashes. ${ }^{1}$ UK data suggest that $10-25 \%$ of truck crashes are related to driver fatigue. ${ }^{2}$ Sixty two (58\%) of 107 US truck drivers involved in a crash attributed it to fatigue and 19 had fallen asleep at the wheel. ${ }^{3}$ In Finland, trailer truck drivers were responsible for $16 \%$ of all two vehicle fatal crashes accidents between 1991 and $1997 .{ }^{4}$

In Israel in recent years, trucks represented $6 \%$ of all vehicles and $28 \%$ of vehicle kilometers travelled, ${ }^{5}$ but truck crashes account for some $20 \%$ of road deaths, ${ }^{6}$ of which more than $90 \%$ occur in the second vehicle. ${ }^{5}$ The high toll, together with accumulating evidence on the role of fatigue, states the case for examining the prevalence of fatigue and its determinants in Israeli truck drivers. Fatigue is highly prevalent in long haul truck drivers covering large regions, ${ }^{7-9}$ and is associated with poor working conditions, ${ }^{10}{ }^{11}$ long work hours, ${ }^{4}{ }^{10}$ chronic illness, ${ }^{4}{ }^{12}$ lack of sleep, and sleep disorders. ${ }^{13}{ }^{14}$ Truck drivers unable to adequately recover from work related fatigue show higher rates of absenteeism from work due to illness. ${ }^{15}$

Data are not readily available on whether risks for fatigue and fatigue related crashes in large countries with long travel distances are present in small countries (for example, Israel which is $467 \mathrm{~km}$ from north to south) with shorter travel distances, and little to no overnight travel or stays away from home, roadside motels, or overnight truck stops. Yet drivers are subject to pressures from employers to complete as many pick-ups and deliveries as possible. Truck driving in Israel is not protected by a powerful union, employees tend to be low on the socioeconomic ladder, and lacks even minimal benefits. Drivers start the workday extremely early, between 0500 and 0600 , before the rapid increase in traffic congestion and outdoor heat, and after extremely long work days, they will arrive home to spend the night with their families.
Our objectives are (1) to report workplace and driving conditions, employer-employee relations, and personal health status-notably sleep quality - in a group of container truck drivers delivering cargo to and from major seaports in Israel and (2) to estimate the association between working conditions and fatigue, falling asleep at the wheel, and previous involvement in truck crashes with casualties.

\section{METHODS}

Of approximately 640 drivers whom we approached at the registration points in the ports of Haifa and Ashdod-the two largest marine distribution ports in Israel-160 (25\%) agreed to be interviewed (see Discussion, Limitations). All drivers delivering or receiving shipments must pass through the registration areas, where two trained researchers (SSE and LF) interviewed them on six separate days in March-April 1997. Field interviews took approximately 10 minutes and were anonymous. We report data on driver characteristics, workplace conditions, employer-employee relations, driving conditions, sleep quality, self-reported fatigue, and history of truck occupational road crashes while working.

The questionnaire was composed of 80 closed ended questions (that is, mostly multiple choice) based on prior information ${ }^{16}$ and three exploratory interviews with Israeli truck drivers. We included a validated questionnaire on sleep quality and disorders used at the Israel Institute of Technology-Technion Sleep laboratory (Haifa, Israel) based on the Pittsburgh Sleep Quality Index, ${ }^{17}$ which measures sleep quality at home and at work. A score of 5 or more on a scale of 10 indicates poor sleep quality and its diagnostic sensitivity reaches approximately $90 \%$ compared with sleep laboratory findings. After review of the questionnaire by

Abbreviations: BMI, body mass index; ILO, International Labor Organization. 
experts on sleep disorders, we pre-tested it on 10 truck drivers.

\section{STATISTICAL ANALYSIS}

We used SAS for Windows (version 8.0) for the statistical analysis. We use $\chi^{2}$ tests for analysis of categorical variables. We calculated Crude odds ratios (OR) and confidence intervals for the outcome variable associated with each predictor variable. We fitted a multiple logistic regression model including potential predictors to estimate adjusted odds ratios (OR), confidence intervals, and $\mathrm{p}$ values for reported fatigue, falling asleep while driving, and involvement in a casualty related crash. The models include the following variables: sleep quality, difficulty finding parking when tired, frequency of driving more than 12 hours/day, employer request to work beyond the legal hour, years of driving experience, body mass index, and reported high blood pressure.

\section{RESULTS}

\section{Driver characteristics}

Table I summarizes personal and occupational information on truck drivers we interviewed. Only 25.9 (15.6\%) worked a regular route. Eighty five (53.1\%) reported driving irregular shifts during the daytime and night-time. Drivers reported being paid by kilometer $(n=1)$, hour $(n=8)$, premium $(n=31)$, salary $(n=52)$, weight delivered $(n=9)$, and other $(n=54)$. One hundred and six $(66.3 \%)$ reported they were regular smokers.

\section{Workplace conditions}

Maximum distances for most trips from the major container port were $<200 \mathrm{~km}$ (125 miles), journeys were relatively short, drivers rarely spent overnight away from home, and overnight sleeping in truck cabins was extremely uncommon. However $61(38.1 \%)$ reported working more than 12 hours during the shift prior to the interview (fig 1), 99 (62\%) reported driving $>12$ hours/day one or more days per week, $48(30 \%)$ reported working $>12$ hours/day almost everyday, and four $(2.5 \%)$ reported working 20 hours or more. The most frequent reason for working $>12$ hours/day was a busy work schedule $(\mathrm{n}=89)$.

Table 1 Characteristics of the Israeli marine port drivers

\begin{tabular}{|c|c|c|}
\hline & n† & $\%$ \\
\hline Mean age, years (range) & $39(21-66)$ & NA \\
\hline \multicolumn{3}{|l|}{ Sex } \\
\hline Male & 159 & 99.4 \\
\hline Female & 1 & 0.6 \\
\hline \multicolumn{3}{|l|}{ Body mass index (BMI)* } \\
\hline$<25$ & 58 & 36.3 \\
\hline $25-29$ & 60 & 37.5 \\
\hline $30-40$ & 23 & 14.4 \\
\hline$>40$ & 1 & 0.6 \\
\hline Regular smokers & 106 & 66.3 \\
\hline \multicolumn{3}{|l|}{ Truck driving experience } \\
\hline$\leqslant 2$ years & 9 & 5.6 \\
\hline $3-9$ years & 36 & 22.5 \\
\hline$\geqslant 10$ years & 115 & 71.9 \\
\hline \multicolumn{3}{|l|}{ Average truck weight } \\
\hline$<16$ tonnes & 5 & 3.1 \\
\hline 16-33.9 tonnes & 26 & 16.3 \\
\hline $34-54$ tonnes & 118 & 73.8 \\
\hline $55+$ & 11 & 6.9 \\
\hline $\begin{array}{l}\text { Salary less than } 4500 \mathrm{NIS} / \text { month } \\
\text { (equivalent to } \$ 16000 / \text { year) } \neq\end{array}$ & 62 & 39 \\
\hline \multicolumn{3}{|c|}{$\begin{array}{l}\text { *No data available for } 18 \text { drivers. } \\
\text { †Absolute numbers unless otherwise specified. } \\
\text { †At } 1995 \text { exchange rate; first quarter of } 1997 \text { US } \$ 1 \text { exchanged for } \\
3.361 \text { NIS (New Israeli Shekel). }\end{array}$} \\
\hline
\end{tabular}

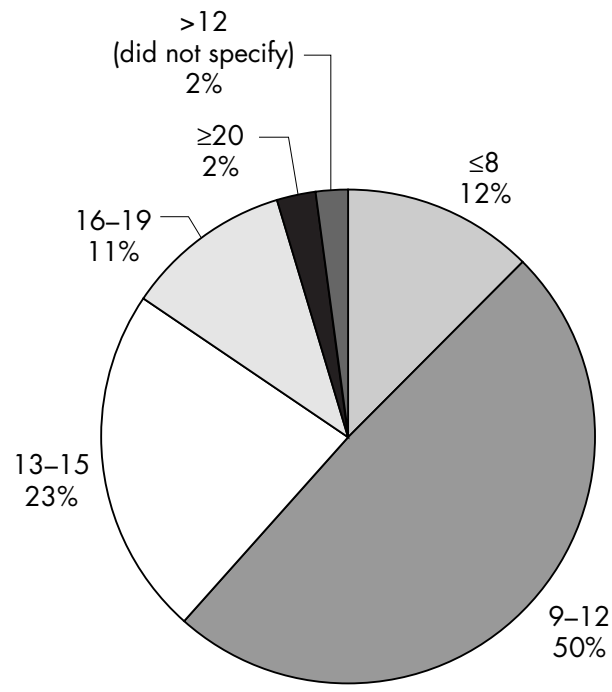

Figure 1 Number of driving hours during shift prior to interview Israeli marine port drivers, 1997.

Forty nine $(30.6 \%)$ drivers worked $>68$ hours during their last working week, of which $36(22.5 \%)$ worked $>84$ hours. Only $18(11.3 \%)$ of the drivers reported working 48 hours/ week or less during the week before the interview.

\section{Employer-employee relations}

Sixty seven $(41.9 \%)$ drivers said that their employer forced them to work beyond the legal 12 hour daily limit. Twenty two drivers $(13.8 \%)$ reported that they were fined or fired for arriving late to a destination. Thirty seven (23.1\%) were verbally rebuked for being late to a destination.

\section{Road conditions}

Only $41(25.6 \%)$ reported never having a problem finding a place to rest when tired on the road. Twenty ( $12.5 \%)$ reported that it was a daily problem, and $96(60 \%)$ as an occasional problem. Drivers reporting frequent difficulty finding rest places were at a higher risk of fatigue compared with drivers with no such difficulty (unadjusted OR 7.8; 95\% CI 2.9 to 21.2).

\section{Medical conditions}

Only $28(17.5 \%)$ of the drivers had a medical check up before being hired by their current employer. Twenty four (15\%) had a body mass index indicating obesity (BMI $>30$ ), a risk for sleep disorders, including sleep apnea. 107 (66.9\%) had one or more of the following health problems: hypertension $(n=8)$, constipation $(n=10)$, gastritis/peptic ulcer $(n=25)$, paresthesias of legs or arms $(\mathrm{n}=38)$, lower back pain $(n=67)$, gluteal pain $(n=23)$, and hemorrhoids $(n=21)$. Sleep quality scores indicated that $34(21.3 \%)$ had moderate to severe sleep quality, of which $16(10.0 \%)$ had indications of severe sleep disturbance. BMI was strongly associated with moderate to severe sleep quality (unadjusted OR 1.2; 95\% CI 1.1 to 1.3 ). Based on these findings, for each 10 unit increase in BMI there was an associated risk of moderate to severe sleep quality of OR 5.078 (unadjusted; 95\% CI 1.8 to 14.2).

\section{Driving conditions and reported fatigue and falling asleep at the wheel}

Sixty three (39.4\%) truck drivers recalled reported having experienced fatigue while driving, of whom 58 (92\%) recalled frequent difficulties finding parking when tired, 37 (59\%) were pressured to work beyond permitted work hour limits, and $52(83 \%)$ drove more than 12 hours/day. 
Fifty $(31.3 \%)$ reported having fallen asleep at the wheel at least once during their entire occupational trucking careers, of whom $17(10.6 \%)$ reported falling asleep at the wheel during the past month. The overwhelming majority of drivers reporting this outcome recalled frequent difficulty finding parking when tired, $(\mathrm{n}=48 ; 96 \%)$, employer demands $(\mathrm{n}=27 ; 54 \%)$, and long work hours $(\mathrm{n}=41 ; 82 \%)$.

Forty drivers $(31.8 \%)$ out of 126 with scores indicating good sleep quality reported suffering from fatigue while driving, compared with eight drivers $(44.4 \%)$ of 18 with slight to moderate sleep quality, and $12(75.0 \%)$ of 16 with severe problems with sleep quality. Thus, although there appears to be a dose-response relation between impairments in sleep quality and fatigue, the majority of drivers with fatigue on the job did not suffer from sleep quality.

There were strong positive associations between falling asleep while driving and moderate to severe sleep quality (OR 2.1 ), frequent difficulty finding parking when tired (OR 14.8), employer demanding the driver to work more than the legal hour limit (OR 2.1), and working more than 12 hours/ day several times a week. But there was an inverse association with driving more than 12 hours/day less than several times a month. (table 2). However after adjustment for the other variables, the predominant predictor of falling asleep while driving is frequent difficulty finding parking when tired (adjusted OR 12.7; $\mathrm{p}=0.001$ ). Overall, the work problems producing the strongest associations with fatigue, falling asleep at the wheel, as well as crashes with injuries/ fatalities (see next section) were seen in the majority of the drivers, without the sleep difficulties seen in the others.

\section{History of prior truck crashes}

One hundred (15.6\%) drivers reported being involved in a crash, of which 33 involved injuries or fatalities. Twenty one (67\% out of 33 ) attributed the reported crash to fatigue and 11 (33\% out of 33$)$ said that the crash was related to sleep problems. Among these 33, many recalled parking problems when tired, $(n=29)$, long working hours at least several times a week $(n=24)$, and employer demands $(n=15)$. For a subgroup of 12 drivers, involvement in a crash with casualties was associated with moderate to severe sleep quality (adjusted OR 2.9; $\mathrm{p}=0.04$ ) and frequent difficulty finding parking when tired (adjusted OR 3.7; $\mathrm{p}=0.05$ ).

\section{DISCUSSION}

Working conditions among the Israeli container truck drivers that we interviewed appear to be severe, despite short travel distances and journey lengths, in addition to the very low frequency of all-night trips. Approximately $38 \%$ of the drivers had worked more than the legal 12 hour daily driving limit one day before interview. A driver starting work at 0500 could log 12 hours by 1700 and 19 hours by midnight, as happened with a small subgroup.

More than 30\% the 160 drivers recalled falling asleep at least once while driving, and $10.6 \%$ had fallen asleep while driving in the month before the interview. Among the drivers, $21.3 \%$ had indications they suffered from sleep disorders. Falling asleep at the wheel and being involved in a crash with casualties was strongly associated with difficulty finding parking and rest stops, an extremely common problem, and poor sleep quality in the subgroup reporting this problem. The finding that fatigue on the job, falling asleep on the wheel, and prior involvement in crashes occurred more often than not without self reported problems with sleep quality suggests that working conditions were the major risk for all three outcomes. Subjective assessment of fatigue underestimated its occurrence as verified by the Pittsburgh Sleep Quality Questionnaire.

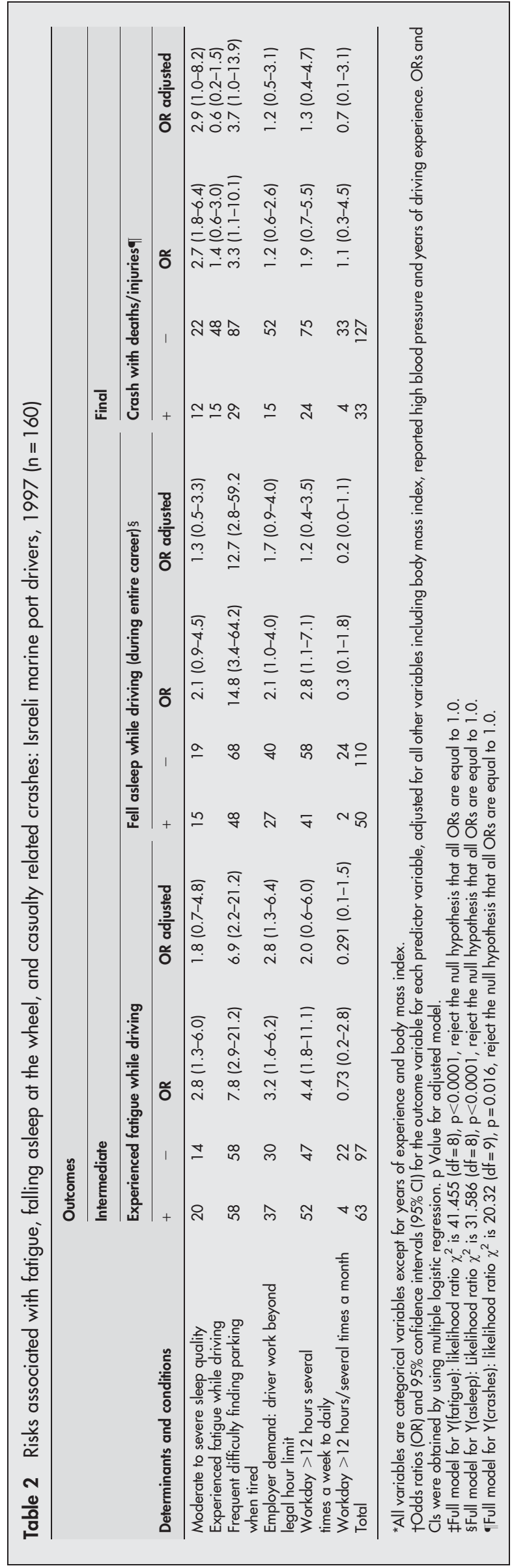




\section{Limitations}

These findings are limited to a "grab sample" type group of marine port container drivers-a relatively select group of truck drivers. As regards selection biases, we surmise we were more likely to interview drivers with more time between loading and unloading and those not afraid of being fined by the police for violations of the permitted daily driving limits. The fact that some interviewees refused to continue to answer questions when asked about their relationship with their employers or their driving histories suggests that we were unable to interview drivers working under the most severe driving conditions.

The rushed working conditions at the registration centers accounted for the high non-response rate $(75 \%)$. We were unable to ascertain the direction, if any, of biases from this high non-response rate, nor rule out recall bias. However the general direction of associations and internal fit between all indicators of work conditions and the three outcomes of increasing severity were strikingly coherent, and consistent with findings reported elsewhere. The positive associations between a heavy work schedule (working several days a week $>12$ hours) and fatigue, falling asleep at the wheel, and crashes, compared with negative associations found between a lighter work schedule and the same outcomes adds internal coherence to the findings.

We suspect that our findings underestimated hazards and risks. Also, so as to not deter interviewees, we did not ask about drink and drug use, which make a non-trivial contribution to one vehicle fatal crashes (a virtually nonexistent outcome for drivers of container trucks in Israel) as well as two vehicle fatal night-time crashes. ${ }^{18}$

Fatigue is generally well correlated with sleep quality. ${ }^{19}$ Previous involvement in a crash with injuries or fatalities was positively associated with moderate to severe impairments in sleep quality as assessed from the Pittsburgh Sleep Quality
Index and negatively associated with answers from a simple query ("do you experience fatigue when driving?"; a far less sensitive question). The simplest and most plausible explanation for this discrepancy is that drivers themselves underestimate, ignore, or deny levels of fatigue associated with increased risk for crashes.

\section{Preventive implications}

The three main occupational determinants of fatigue are reported to be length of work day, duration of time spent away from work for rest, and work-rest pattern in a 24 hour day. ${ }^{20}$ Israeli container drivers are at risk for work related fatigue, falling asleep at the wheel, involvement in crashes resulting from difficulty finding rest stops, employer pressures, and long work hours-even in the majority without sleep quality impairments.

In 1988, Hertz reported that $41 \%$ of heavy truck crashes occurred after 16 hours or more on duty. ${ }^{21}$ Among Australian long haul drivers, $51 \%$ exceeded 14 hours of driving, $40 \%$ of dangerous events occurred among those who reported sleeping less than 6 hours, and crash risks were 2.5 times greater after 14 hours of work compared with less than 10 hours. ${ }^{22}$ Our findings show that Israeli short haul truckers with journeys usually much shorter than $200 \mathrm{~km}$ (approximately 120 miles) and workdays exceeding 12 hours/day had increased risks for falling asleep at the wheel (unadjusted OR $2.8 ; \mathrm{p}<0.05$; adjusted $1.2 ; \mathrm{p}>0.05$ )

The high toll of road deaths from truck crashes and the adverse working conditions of many Israeli truck drivers state the case for preventive measures. The fact that self-reported fatigue and falling asleep at the wheel occurred in most drivers without problems in sleep quality points to the importance of the work conditions-above all, problems with finding parking and rest spots, along with long hours and employer pressure, as the most important determinants of

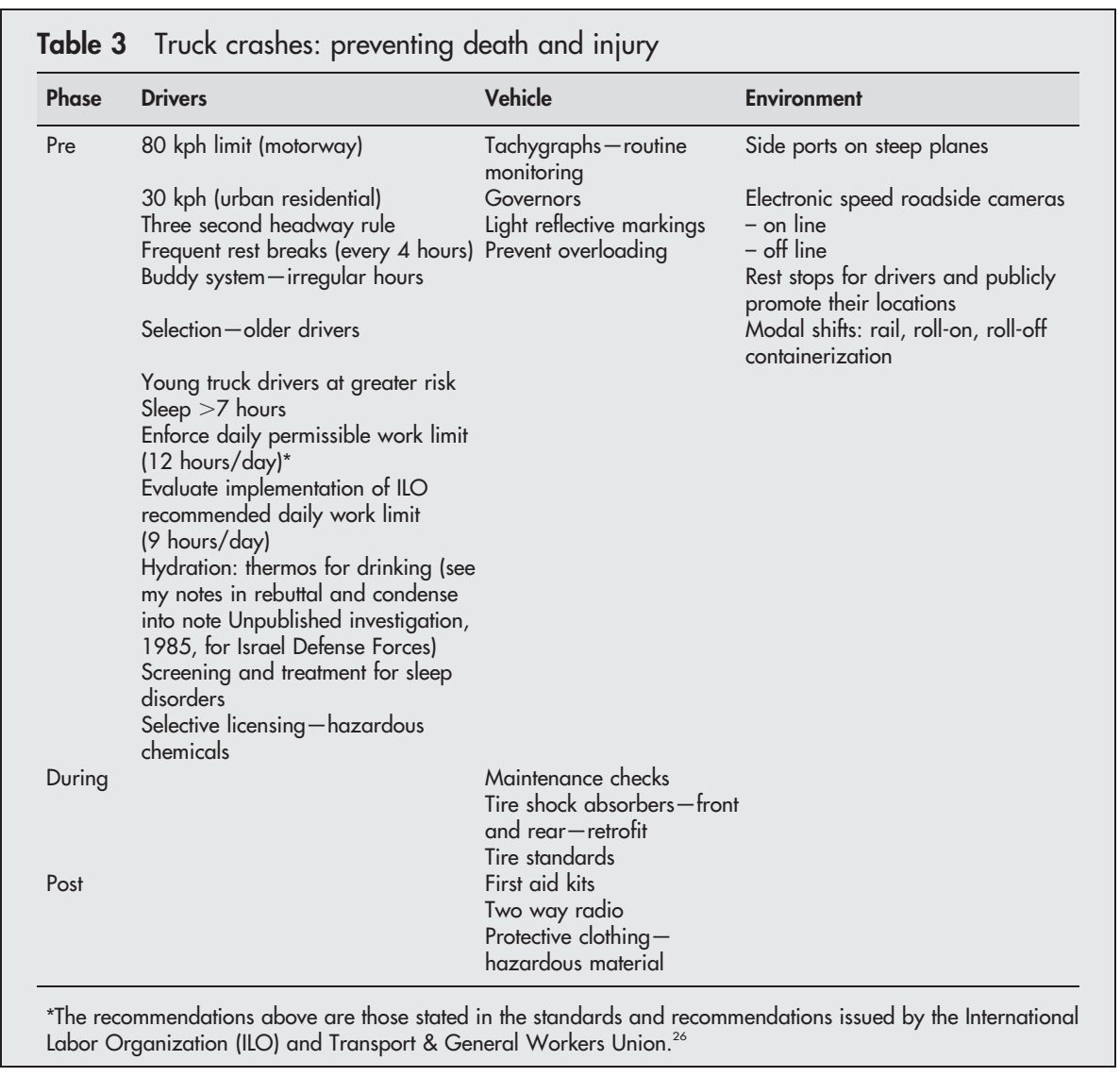


fatigue. But the fact that many crashes with injuries occurred without fatigue suggests that interventions based on targeting fatigue and work conditions alone cannot be expected to prevent all truck related deaths and injuries. Therefore, other measures, including speed control and shifts to alternative modes of transportation are required.

We present a list of preventive measure in the framework of the Haddon Grid (table 3), and a model (fig 2, which can be found at http://www.injuryprevention.com/supplemental) that examines reinforcing interrelations between job stressors, individual host risks, non-work risks, and individual acute reactions and the mitigating effects of interventions. The destructive combination of mass and velocity, the role of raised speed limits in increasing deaths from truck crashes, and the success of speed cameras in the UK and Australia in reducing road deaths state the case for their benefits, ${ }^{23}$ with or without measures specifically targeting fatigue risks. Additional measures include regulatory monitoring of tachygraphs (for speed and work hours), and industry wide screening of truckers for sleep disorders and poor sleep quality to identify this high risk group. Such screening for high risk individuals should not serve as an alternative to improving work conditions.

The dominant role of searching for parking and rest stops as a risk for fatigue, falling asleep at the wheel, and crashes states the case for roadside rest stops. Enforcement of International Labor Organization (ILO) ${ }^{24}$ standards-would curb many dangerous practices. These standards specify a ceiling of nine hours of work per day or 48 hours per week, which is substantially less than 12 hours/day and 68 hours/ week permitted by Israeli regulations. The ILO also specifies not driving for more than four hours without rest.

We recommend higher base wages and doing away with incentive productivity premiums for trips completed. Low base pay and premiums, promoting travel at higher speeds, increase the risks. Because interventions targeted at risks for fatigue and falling asleep at the wheel and crashes will not by themselves prevent all truck related injuries, there is a strong case for "out of the box" solutions based on modal shifts to rail shipment, and roll-on/roll-off rail truck container systems.

Our findings indicate that fatigue, falling asleep while driving, and fatal crashes among truck drivers can occur without the so-called tyranny of long travel distances and overnight travel. Furthermore, involvement in crashes can occur without fatigue or problems with sleep. The economic profit margins and competitiveness of truck transport create pressures for maximum amounts of mass moved from source to destination in a minimum of time through use of double trailers, maximum loads, higher travel speeds, and long working hours.

Israel's trucker union is weak and only a small minority of interviewed truckers belonged to it. Therefore policy change must come from government agencies. The US Department of Transportation Truck and Bus Safety Summit ${ }^{25}$ ranked fatigue as the first priority out of 17 target issues.

We hypothesize that reducing fatigue related truck crashes, together with speed control, would save many lives. ${ }^{26}{ }^{27}$ But experimental intervention is required to determine which combination of interventions drawn from ILO standards and the Haddon Grid give the largest, swiftest, and most cost effective reductions in the toll of death and injury.

\section{Authors' affiliations}

S Sabbagh-Ehrlich, E D Richter, Hebrew University-Hadassah School of Community Medicine and Public Health, Unit of Occupational and Environmental Medicine, Injury Prevention Center, Jerusalem, Israel L Friedman, The Social Policy Research Institute, Skokie, IL, USA

\section{Key points}

- Trucks represent $6 \%$ of all vehicles, but account for $20 \%$ of road deaths in Israel even though travel distances are short $(<200 \mathrm{~km})$ and overnight travel is uncommon.

- Long work hours, falling asleep at the wheel, and employer pressures to work long hours were common in port container truck drivers. Involvement in crashes with casualties was associated with poor sleep quality and frequent difficulty finding parking when tired.

- Fatigue occurred in many drivers without sleep problems, and many crashes occurred without fatigue. Self assessment was an insensitive indicator of fatigue.

- Prevention requires measures to reduce work stresses, screening drivers, speed control, and modal shiftseven in a small country without "the tyranny of distance"

\section{REFERENCES}

1 Ladou J. The health of truck drivers. In: CarlZenz, ed. Occupational medicine-principles and practical application, 2nd edn, 1988:958-69.

2 Maycock G. Driver sleepiness as a factor in car and HGV accidents. Transport Research Laboratory, Report 169. 1995:1-39.

3 National Transportation Safety Board Analysis-Safety Study Vol 1. 1995: 1-90.

4 Hakkanen $\mathrm{H}$, Summala $\mathrm{H}$. Fatal traffic accidents among trailer truck drivers and accident causes as viewed by other truck drivers. Accid Anal Prev 2001;33:187-96.

5 Israel Central Bureau of Statistics. Road Accidents with Casualties Annual Reports, and Transport Statistics Quarterly Reports. Jerusalem, Israel: 2001.

6 Richter ED, Barach P, Friedman LS, et al. Raised speed limits, speed spillover, case fatality and road deaths in Israel: a five-year follow-up. Am J Pub Health 2004;94:568-74.

7 Stoohs RA, Bingham L, Itoi A, et al. Sleep and sleep-disordered breathing in commercial long-haul truck drivers. Chest 1995;107:1275-82

8 Hakkanen $\mathrm{H}$, Summala $\mathrm{H}$. Sleepiness at work among commercial truck drivers. Sleep 2000;23:49-57.

9 Mitler MM, Miller JC, Lipsitz JJ, et al. The sleep of long-haul truck drivers. N Engl J Med 1997; 337:755-61.

10 Beilock R. Schedule-induced hours-of-service and speed limit violations among tractor-trailer driver. Accid Anal Prev 1995;27:33-42.

11 Adams-Guppy J, Guppy A. Truck driver fatigue risk assessment and management: a multinational survey. Ergonomics 2003:46:763-79.

12 Laberge-Nadeau C, Dionne G, Ekoe JM, et al. Impact of diabetes on crash risks of truck-permit holders and commercial drivers. Diabetes Care 2000;23:612-17

13 Aldrich MS. Automobile accidents in patients with sleep disorders. Sleep 1989; 12:487-94.

14 Horne JA, Reyner LA. Driver sleepiness. J Sleep Res 1995;4:23-9.

15 de Croon EM, Sluiter JK, Frings-Dresen MH. Need for recovery after work predicts sickness absence: a 2-year prospective cohort study in truck drivers $J$ Psychosom Res 2003:55:331-9.

16 Braver ER, Preusser CW, Preusser DF, et al. Long hours and fatigue: a survey of tractor-trailer drivers. J Public Health Policy 1992;13:341-66.

17 Buysse DJ, Reynolds CF, Monk TH, et al. The Pittsburgh Sleep Quality Index: a new instrument for psychiatric practice and research. Psychiatry Res 1989:28:193-213.

18 Richter ED, Meltzer U, Bendov G, et al. Alcohol levels in drivers and pedestrians killed in road accidents in Israel. Int J Epidemiol 1986;15:272-3

19 Lavidor M, Weller A, Babkoff $\mathrm{H}$. How sleep is related to fatigue. Br J Health Psychol 2003;8:95-105.

20 Transport \& General Workers Union (TGWU). Fatigue-the hidden killer on our roads. TGWU Publications, 1994

21 Hertz RP. Tractor-trailer driver fatality: the role of non-consecutive rest in a sleeper berth. Accid Anal Prev 1988;20:431-9.

22 Arnold PK, Hartley LR, Corry A, et al. Hours of work, and perceptions of fatigue among truck drivers. Accid Anal Prev 1997;29:471-7.

23 Evans L. Traffic safety. Bloomfield Hills MI: Science Serving Society, 2004.

24 International Labor Organization. Hours of Work and Rest Periods (Road Transport) Recommendation 161, Conference 65. Adopted in 1979

25 US Department of Transportation, Federal Highway Administration. The top truck and bus safety issues, 1995. Available at http://www.tfhrc.gov/ pubrds/summer95/p95su20.htm (accessed 18 January 2005)

26 Ben-David G, Neeman V, Apter J, et al. Trends in deaths and injury from truck crashes: Israel (4th International Conference on Traffic Safety, Tel Aviv). In: Hakkert AS, ed. Israel National Road Safety Authority, 1997:620-33.

27 Soudry A, Slater PE, Richter ED. Military and civilian motor vehicle crashes, Israel. Travel Med Int 1984;2:250-4. 